\title{
Prediction and prevention of hypertensive disorders of pregnancy: a methodological mistake
}

Hypertension Research (2017) 40, 788; doi:10.1038/hr.2017.33; published online 9 March 2017

I was interested to read the paper by Ohkuchi et al. ${ }^{1}$ published in Hypertens Res Jan 2017. The most common classifications of hypertensive disorders of pregnancy are chronic hypertension, gestational hypertension, preeclampsia (PE) and superimposed PE. The most successful translational research model for explaining the development of PE is the angiogenic/angiostatic balance theory, which involves soluble fms-like tyrosine kinase-1, placental growth factor and soluble endoglin. The aim of the authors was to predict earlyonset $\mathrm{PE}$ in the beginning of the third trimester. In addition, the authors suggested that an onset threshold or a serial approach appeared to be clinically useful for predicting the imminent onset of PE. The study suggested that onset occurring $<4$ weeks after blood sampling in the second or early-third trimesters may be predictable because the observed positive likelihood ratio was $>10$ and the positive predictive value was $>20 \%{ }^{1}{ }^{1}$

However, this result has nothing to do with prediction. First, positive likelihood ratio and positive predictive values are estimates that are used to evaluate the diagnostic accuracy of a single test compared to a gold standard. Moreover, for prediction studies, we need data from two different cohorts or at least from one cohort divided into two to first develop a prediction model and subsequently validate it. Misleading results are generally the main outcome of research that fails to validate its prediction models. ${ }^{2-6}$

Finally, in prediction studies, we must assess the interactions between important variables. Final results can be impacted markedly when qualitative interactions are present. ${ }^{2-6}$ This means that most of the time, without assessing the interaction terms, prediction studies will mainly produce misleading messages.

\section{CONFLICT OF INTEREST}

The author declares no conflict of interest.

Siamak Sabour ${ }^{1,2}$

${ }^{1}$ Safety Promotion and Injury Prevention Research Center, Shahid Beheshti
University of Medical Sciences, Tehran, Iran and ${ }^{2}$ Department of Clinical Epidemiology, School of Health, Shahid Beheshti University of Medical Sciences, Tehran, Iran

E-mail: s.sabour@sbmu.ac.ir

1 Ohkuchi A, Hirashima C, Takahashi K, Suzuki H, Matsubara S. Prediction and prevention of hypertensive disorders of pregnancy. Hypertens Res 2017; 40: 5-14.

2 Rothman KJ, Sander G, Timothy LL. Cohort studies. In Rothman KJ (ed.). Modern Epidemiology, 3rd edn. Lippincott Williams \& Wilkins: Baltimore, MD, 2008, pp 79-85.

3 Sabour S. Prediction of preterm delivery using levels of VEGF and leptin in amniotic fluid from the second trimester: prediction rules. Arch Gynecol Obstet 2015; 291: 719.

4 Sabour S. Prediction of 3-dimensional pharyngeal airway changes after orthognathic surgery: a methodological issue. Am J Orthod Dentofacial Orthop 2015; 147: 8.

5 Sabour S. Obesity predictors in people with chronic spinal cord injury: a common mistake. J Res Med Sci 2014; 19: 80.

6 Sabour S, Ghassemi F. Predictive value of confocal scanning laser for the onset of visual field loss. Ophthalmology 2013; 120: e31-e32. 